\title{
Spin Wave Diffraction and Perfect Imaging of a Grating
}

\author{
S. Mansfeld,${ }^{1}$ J. Topp,${ }^{1}$ K. Martens,${ }^{1}$ J. N. Toedt, ${ }^{1}$ W. Hansen, ${ }^{1}$ D. Heitmann,${ }^{1}$ and S. Mendach ${ }^{1, *}$ \\ ${ }^{1}$ Institut für Angewandte Physik und Mikrostrukturforschungszentrum, \\ Universität Hamburg, Jungiusstrasse 11, D-20355 Hamburg, Germany
}

(Dated: November 2, 2018)

\begin{abstract}
We study the diffraction of Damon-Eshbach-type spin waves incident on a one-dimensional grating realized by micro slits in a thin permalloy film. By means of time-resolved scanning Kerr microscopy we observe unique diffraction patterns behind the grating which exhibit replications of the spin-wave field at the slits. We show that these spin-wave images, with details finer than the wavelength of the incident Damon-Eshbach spin wavelength, arise from the strongly anisotropic spin wave dispersion.
\end{abstract}

The possibility to use spin waves to excite, store and retrieve electric signals and to perform logical operations relies on the ability to manipulate spin wave propagation. In patterned, thin ferromagnetic films spin waves with gigahertz frequencies and wavelengths from several hundreds of nanometers up to millimeters can be confined [18, focused and guided 9 14]. An intriguing possibility to guide spin waves in unpatterned films, lies in their anisotropic dispersion. The dispersion of dipole-dominated spin waves in thin ferromagnetic films strongly depends on their propagation direction $\vec{k}$ with respect to an in-plane magnetic field $\vec{H}_{0}$ giving rise to Damon-Eshbach modes with $\vec{k}_{D E} \perp \vec{H}_{0}$, BackwardVolume modes with $\vec{k}_{B V} \| \vec{H}_{0}$, or a combination of both. This anisotropy in the dispersion of spin waves [15-17. has recently been studied theoretically 18 and experimentally [19 21] as a novel way to manipulate spin wave propagation.

In this letter we demonstrate that the anisotropic spinwave dispersion enables perfect imaging with spin waves. We present and discuss time resolved scanning Kerr microscopy (TR-SKM) 22, 23, data on the diffraction of planar Damon-Eshbach spin waves on a one-dimensional grating realized by micrometer sized slits in a permalloy film. Behind this grating, we observe a unique diffraction pattern which arises from the interference of Damon-Eshbach and Backward-Volume type spin waves and their inherent strongly anisotropic dispersion. We show that this diffraction pattern produces images of the spin wave field at the slits. The resolution of these images is not limited by the wavelength of the incident spin wave, as it is the case in isotropic media [24, but deviations from a perfect image occur solely due to spin wave damping and due to the finite curvature of the dispersion's iso-frequency line in $k$ space. In that sense, our spin wave images represent a spin-wave analogue to sub-wavelength resolution concepts with anisotropic media in optical metamaterials [25, 26]. In addition, we demonstrate that the position of the spin wave images behind the slit array can be tuned by manipulating the spin-wave dispersion via the excitation frequency and the external magnetic field. This enables us to create tailormade spin-wave fields in an unpatterned ferromagnet film

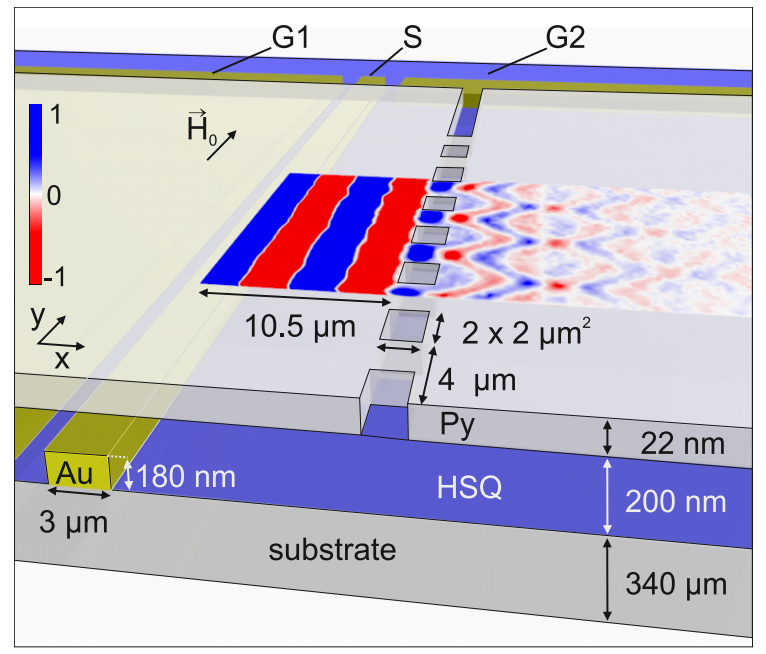

FIG. 1. FIG. 1 (color online): Sketch of the experimental arrangement. S: Signal line, G1, G2: Ground lines of the coplanar wave guide. An exemplary TR-SKM phase image of the spin wave field taken at $\mu_{0} H_{0}=10 \mathrm{mT}$ and $f=4180 \mathrm{MHz}$ is overlaid as a color density plot on a part of the Permalloy film.

with sub-micron resolution.

The experimental arrangement is sketched in Fig. 1. The investigated sample is a $22 \mathrm{~nm}$ thick Permalloy film prepared on top of a coplanar wave guide (CPW) structure. The CPW was prepared on a $340 \mu \mathrm{m}$ thick $\operatorname{GaAs}(100)$ substrate and consists of a $3 \mathrm{~nm}$ thick adhesive layer of chromium and a $180 \mathrm{~nm}$ thick layer of gold. It features a $3 \mu \mathrm{m}$ wide signal line (S) separated by $2 \mu \mathrm{m}$ gaps from the ground lines $(\mathrm{G} 1, \mathrm{G} 2)$. G2 exhibits a $150 \mu \mathrm{m}$ wide gap at the position of the Permalloy film; this guarantees the injection of well defined planar spin waves from the signal line (S). An approximately $200 \mathrm{~nm}$ thick layer of hydrogen silsesquioxane was applied on top of the wave guide acting as an insulator between the wave guide and the Permalloy film. The film has a rectangular shape with a length of $l=150 \mu \mathrm{m}$ perpendicular and $w=120 \mu \mathrm{m}$ parallel to the CPW. At a distance of $10.5 \mu \mathrm{m}$ from the center of the signal line (S), the Permalloy film features an one-dimensional grating realized by 
13 square-shaped holes with a side length of $2 \mu \mathrm{m}$ and a periodicity of $p=4 \mu \mathrm{m}$. The permalloy film was prepared using vacuum thermal evaporation in combination with electron beam lithography and lift off technique.

TR-SKM is used to examine the spin wave propagation in the Permalloy film. A pulsed Titan-Sapphire laser with a wavelength of $800 \mathrm{~nm}$ and a repetition rate of $76 \mathrm{MHz}$ is focused onto the sample to probe the magnetization component perpendicular to the Permalloy film via the magneto optical Kerr effect. The laser focus has a width of approximately $550 \mathrm{~nm}$ and is scanned over the sample in $250 \mathrm{~nm}$ steps. Spin waves are excited in the film by passing a continuous microwave through the CPW. The customized microwave synthesizer (ITS 9200) used to excite the spin waves is phase locked to the repetition rate of the laser. By tuning the phase offset between laser and microwave synthesizer, the spin-wave field in the sample can be measured phase dependently. This enables us to image propagating spin wave fields with a temporal resolution of a few Picoseconds. Initially, we have optimized the set up shown in Fig. 1 with experiments on homogenous unpatterned films and found that, except for edge effects which extent less than $15 \mu \mathrm{m}$ from the edges of the film, the CPW emits planar spin waves with wave vectors tuneable up to $k=4.5 \mu \mathrm{m}^{-1}$. The color encoded plot overlaid onto the sample sketch in Fig. 1 represents an exemplary measurement. As indicated by the arrow, the external magnetic field is oriented parallel to the CPW. All magnetic fields quoted in the following have been applied after applying a saturation field of $80 \mathrm{mT}$ in y direction and ramping down to $0 \mathrm{mT}$. A Damon-Eshbach type spin wave is excited at the signal line $(\mathrm{S})$ and propagates towards the grating. At the grating the spin wave is partly reflected and partly transmitted. In the following we investigate the transmitted part and its unique interference patterns. We show that these patterns produce images of the grating at distinct positions.

Let us first concentrate on three TR-SKM images depicted on the left hand sides of Figs. 2(a),(c),(e) and measured at $\mu_{0} H_{0}=20 \mathrm{mT}$ with an excitation frequency of $f_{1}=4180 \mathrm{MHz}, f_{2}=4636 \mathrm{MHz}$ and $f_{3}=5092 \mathrm{MHz}$, respectively. These images show the propagating spin wave field at a defined phase of the excitation, i.e., they represent snap shots of the spin wave field for a certain point in time. In the following they are referred to as phase plots. The holes in the Py-film defining the grating are marked by dashed black lines. For all frequencies we observe distinct interference patterns which are periodic in propagation direction ( $x$ direction) and along the grating ( $y$ direction). With increasing frequency we find that the period in propagation direction gets smaller while the period along the grating is fixed. This is also reflected in the corresponding spatial Fourier spectra of the area behind the grating, which are shown on the right hand side of Figs. 2(a),(c),(e). We observe well defined inten-

$$
\mu_{0} H_{0}=20 \mathrm{mT}
$$
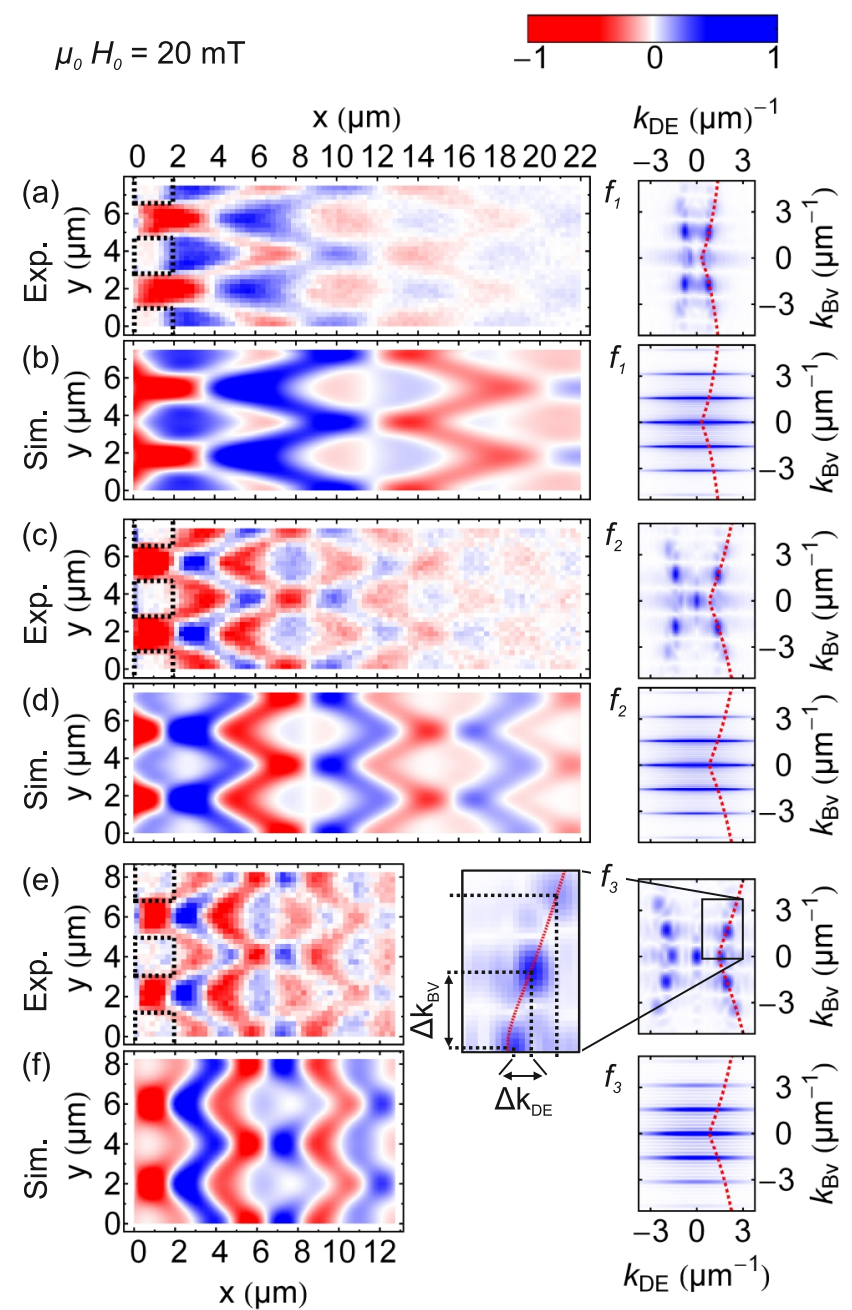

FIG. 2. FIG. 2 (color online): (a), (c) and (e) TR-SKM phase image of the spin-wave field (left side) behind the grating and corresponding Fourier spectra (right side) measured at $H_{0}=20 \mathrm{mT}$ with $f_{1}=4180 \mathrm{MHz}, f_{2}=4636 \mathrm{MHz}$, and $f_{3}=$ $5092 \mathrm{MHz}$, respectively. The holes in the Py-film defining the grating are marked by dashed black lines in the TR-SKM images on the left side. (b), (d) and (f) Calculated spin-wave fields for $f_{1}=4180 \mathrm{MHz}, f_{2}=4636 \mathrm{MHz}$ and $f_{3}=5092 \mathrm{MHz}$ (left side) and Fourier spectra of an analytical approximation of the slit array. Here $k_{D E}$ and $k_{B V}$ are, respectively, spinwave vector components in $\mathrm{x}$ direction and in $\mathrm{y}$ direction. The red dashed lines plotted in all Fourier spectra mark the theoretical DIFL.

sity maxima in the Fourier spectra which correspond to the spin-wave modes contributing to the respective interference pattern. Additionally, we find peaks for $k_{D E}=0$ and $k_{B V}=0$, which are not related to the propagating spin-wave field. The Backward-Volume components $\left|k_{B V}\right|$ of the observed modes correspond to the grating period in $y$ direction $(p=4 \mu \mathrm{m})$ and are the same for all excitation frequencies $\left(\left|k_{B V 0}\right|=0,\left|k_{B V 1}\right|=1.65 \mu \mathrm{m}^{-1}\right.$, $\left.\left|k_{B V 2}\right|=3.30 \mu \mathrm{m}^{-1}\right)$. In contrast to this, the related 
Damon-Eshbach components $\left|k_{D E}\right|$ which correspond to the period in propagation direction increase with increasing frequency. They are imposed by the anisotropic dispersion law of spin waves in the Py film, since only wave vectors fullfilling this dispersion law can propagate away from the grating. Therefore, the experimental Fourier spectra shown in Figs. 2(a),(c),(e) give the convolution of the grating's Fourier spectrum with the dispersion's iso-frequency line in $k$ space (DIFL) at different frequencies (The DIFL is also referred to as 'slowness curve' in recent literature [18, 21]). Indeed, we find that the observed maxima fit very well with calculated DIFLs (red dashed lines) obtained with an analytical model by Guslienko et al. 27] for a $22 \mathrm{~nm}$ thin ferromagnetic film with a saturation magnetization of $\mu_{0} M_{s}=0.94 \mathrm{mT}$.

A first-principal calculation of the diffraction pattern would be an extremely challenging task. It would require a detailed knowledge of the static spin boundary condition at the edges of the holes and the arising static and dynamic magnetization pattern at the grating in an external magnetic field. However, we found that with a simple analytical ansatz we can well reproduce the observed interference pattern and get a deep insight into the microscopic spin wave propagation: We use an array of 13 intensity distributions $s(\vec{r})=S_{0} \cdot \operatorname{Exp}\left(-2 \vec{r}^{2} \mu \mathrm{m}^{-2}\right)$ spaced $4 \mu \mathrm{m}$ apart as an approximation of the spin-wave distribution at the grating. Phase and amplitude of all modes excited by the diffraction are calculated by taking the Fourier spectrum of this intensity distribution. To obtain those modes of the spectrum which can propagate in the Permalloy film, we convolute the Fourier spectrum of the grating with the calculated DIFLs. This is illustrated in the right hand sides of Figs. 2(b),(d),(f). By superimposing all, in this way identified propagating modes, with the correct amplitude and phase in real space, we calculate the spin-wave interference patterns shown on the left hand sides of Figs. 2(b),(d) and (f). We find a good agreement with the corresponding experimental data shown in Figs. 2(a),(c),(e). Small deviations in the details of the spin-wave patterns are due to the simple approximation we use for the spin-wave distribution at the slit array. Note that it is not sufficient to assume point sources to obtain a good agreement between calculation and experiment.

Figure 3(a) shows an amplitude plot which was measured at $f_{2}=4636 \mathrm{MHz}$ and $H_{\text {ext }}=20 \mathrm{mT}$ and corresponds to the phase plot shown in Fig. 2(c). To obtain the amplitude plot, we scanned through a complete phase period and measured the maximum deflection of the magnetization for all spatial points in the measured field. We find distinct areas with high amplitudes (blue color) and areas with amplitudes near zero (white color). The sharp features in the pattern, e.g. the white lines with a small width of approximately $700 \mathrm{~nm}$ compared to the incident Damon-Eshbach wavelength of $6.3 \mu \mathrm{m}$, indicate the excitation of high $k_{x}$ and $k_{y}$ components at
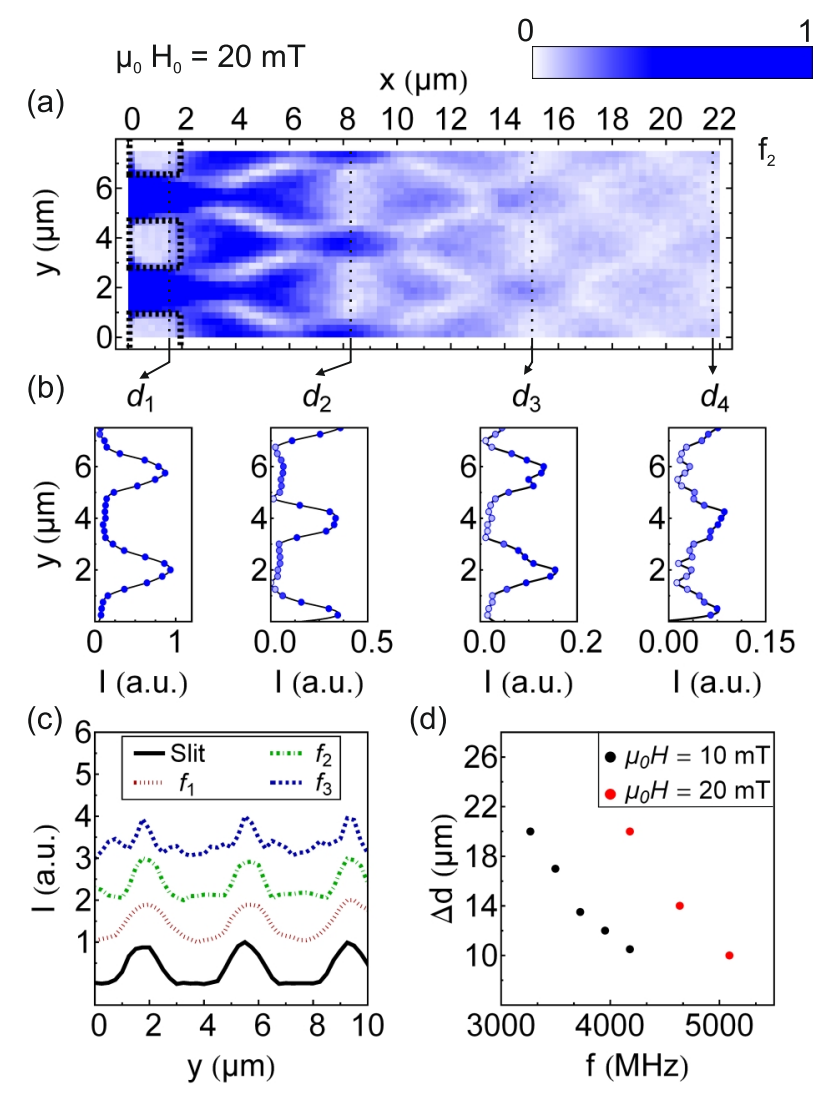

(d)

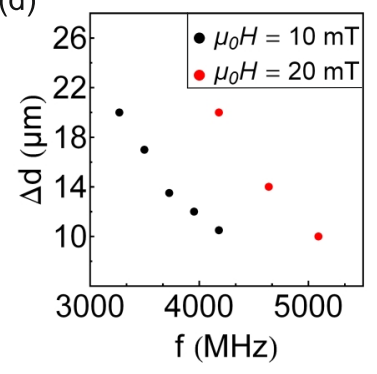

FIG. 3. FIG. 3 (color online): (a) TR-SKM amplitude image of the spin-wave field at $H_{0}=20 \mathrm{mT}$ and $f_{2}=4636 \mathrm{MHz}$ corresponding to the left side of Fig. 2(c). The holes in the Py film defining the grating are marked by thick dashed black lines. (b) Cross sections along the thin dashed black lines at $d_{1}, d_{2}, d_{3}$ and $d_{4}$. (c) Cross sections along the first image line measured at $f_{1}=4180 \mathrm{MHz}, f_{2}=4636 \mathrm{MHz}$ and $f_{3}=5092 \mathrm{MHz}$. (d) Distance between grating and image line $\Delta d$ at $\mu_{0} H_{0}=10 \mathrm{mT}$ (black dots) and $\mu_{0} H_{0}=20 \mathrm{mT}$ (red dots).

the grating.

Most interestingly, the amplitude distribution at the grating $\left(d_{1}=1.50 \mu \mathrm{m}\right)$ is replicated at equidistant $x$ values $d_{2}=8.25 \mu \mathrm{m}, d_{3}=15.00 \mu \mathrm{m}$, and $d_{4}=21.75 \mu \mathrm{m}$ where every other replication is shifted in $y$ direction by half of the grating period $p$. Figure 3(b) illustrates the replications at the respective $x$ values. They represent spin-wave images of the grating cross-section as will be explained in the following.

In general, to obtain a perfect image of the grating cross-section (i) all Fourier components present at the grating cross-section have to reach the respective image lines and (ii) they have to be superimposed with the right phase and with the right weighting of amplitudes. As visible from the Fourier spectra shown on the right hand side of Figs. 2(c) and (d), condition (i) is fulfilled because the DIFL (dashed red line) crosses all grating diffraction or- 
ders which exhibit a considerable intensity, i.e. all Fourier components necessary to obtain an image of the grating cross-section can propagate in our film. Condition (ii) is fulfilled due to the almost linear shape of the DIFL in this wave vector regime and frequency regime. Due to this linearity, the spacing between Fourier components in cross-sectional direction ( $y$ direction), which is given by the reciprocal lattice vector $\Delta k_{B V}=2 \pi / p$ of the grating, leads to an equidistant spacing of the Fourier components in propagation direction with $\Delta k_{D E}=\gamma \Delta k_{B V}$ and $\gamma$ being the slope of the DIFL (cf. zoom in of the Fourier spectrum in Fig. 2(e)). As a result, beating patterns form in real space exhibiting image lines at periodic distances $\Delta d=2 \pi / \Delta k_{D E}=p / \gamma$, at which the phases of all Fourier components correspond to the grating crosssection. Furthermore, at half way between these image lines additional image lines exist, where the phases of all Fourier-components are shifted by $180^{\circ}$ compared to the grating cross-section, leading to an image shift in $y$ direction by $p / 2$. Since the cross-section of the spin-wave field at all $x$ positions inside the slits is imaged as explained above, we in fact observe two-dimensional images of the slits in Fig. 3(a).

Since the slope $\gamma$ of the DIFL changes with frequency $f$ (cf. Figs. 2(a),(c),(e)) and magnetic field $H_{0}$, we can tailor the period of the image lines $\Delta d=p / \gamma$, i.e. their position in the film, via $f$ or $H_{0}$. As shown in Fig. $3(\mathrm{~d})$, we find that $\Delta d$ increases with increasing magnetic field and decreasing frequency. With the parameters of our experiment we obtain values between $10 \mu \mathrm{m}$ and $20 \mu \mathrm{m}$.

Figure 3(c) compares how the spin-wave cross-section at $d_{1}$ is imaged in the respective image lines for $f_{1}=$ $4180 \mathrm{MHz}, f_{2}=4636 \mathrm{MHz}$ and $f_{3}=5092 \mathrm{MHz}$ at $\mu_{0} H_{0}=20 \mathrm{mT}$. While the image quality at $f_{1}$ and $f_{2}$ is comparable, the shorter spin wave damping length at $f_{3}$ leads to a reduction in image quality. In measurements on a $20 \mathrm{~nm}$ thick Py film without grating we found that the 1/e amplitude damping length decreases from approximately $17 \mu \mathrm{m}$ to approximately $5 \mu \mathrm{m}$ if we increase the excitation frequency from $3800 \mathrm{MHz}$ to $5000 \mathrm{MHz}$. Besides the spin wave damping, which degrades the quality of the images also with increasing distance from the grating (cf. Fig. 3(b)), deviation from a perfect image occur due to the finite curvature of the DIFL which smears out the $x$ position of the image lines. Note that, as visible at $d_{2}=8.25 \mu \mathrm{m}$ in Fig. 3(b), this smearing out can also lead to a reduction of the imaged slit width compared to the slit width in the object at $d_{1}=1.50 \mu \mathrm{m}$.

Aside from the perfect spin-wave images presented here another consequence of the linear DIFL is the formation of caustic spin wave beams with direction given by the normal of the DIFL as discussed in recent literature 1921 . While these caustic beams do not appear in the diffraction pattern of our grating structure, we indeed observe caustic beams in a double-slit structure (not shown) which was prepared in accordance to the grat- ing structure. The appearance of caustic beams in the double-slit structure is most probably due to its smoother Fourier spectrum compared to the grating. Interestingly, we found one single slit's spin wave image at the crossing of the caustic beams. This reflects the fact that the crossing points of the caustic beams coincide with the image lines discussed above.

In conclusion, we investigated the diffraction of planar Damon-Eshbach spin waves incident on a grating and on a double slit by means of TR-SKM measurements. We found that due to the linearly shaped DIFL spin-wave images of the slits form behind the structures. The quality of these images is only limited by spin wave damping and the deviation of the DIFL from a linear shape. Furthermore, we demonstrated that the image position in the film behind the periodic structures can be tuned by changing the slope of the DIFL via the excitation frequency or external magnetic field. These findings enable new concepts for controlled spin-wave confinement and manipulation in unpatterned ferromagnetic films via adjacent slit arrays, e.g. to realize novel spin-wave filters or logic devices.

We acknowledge major contributions to the setting up of our TR-SKM by Jan Podbielski and Andreas Krohn as well as fruitful discussions with Andreas Rottler and Stephan Schwaiger and financial support by the DFG via SFB 668, SFB 508, GrK 1286, and by the City of Hamburg via the Cluster of Excellence Nano-Spintronics.

smendach@physnet.uni-hamburg.de

[1] J. Jorzick, S. Demokritov, C. Mathieu, B. Hillebrands, B. Bartenlian, C. Chappert, F. Rousseaux, and A. Slavin, Physical Review B 60, 15194 (1999)

[2] M. Buess, R. Höllinger, T. Haug, K. Perzlmaier, U. Krey, D. Pescia, M. Scheinfein, D. Weiss, and C. Back, Physical Review Letters 93, 077207 (2004).

[3] C. Bayer, J. Jorzick, B. Hillebrands, S. Demokritov, R. Kouba, R. Bozinoski, A. Slavin, K. Guslienko, D. Berkov, N. Gorn, and M. Kostylev, Physical Review B 72, 064427 (2005)

[4] K. Perzlmaier, M. Buess, C. Back, V. Demidov, B. Hillebrands, and S. Demokritov, Physical Review Letters 94, $057202(2005)$

[5] I. Neudecker, K. Perzlmaier, F. Hoffmann, G. Woltersdorf, M. Buess, D. Weiss, and C. Back, Physical Review B 73, 134426 (2006)

[6] J. Podbielski, F. Giesen, and D. Grundler, Physical Review Letters 96, 167207 (2006)

[7] S. Mendach, J. Podbielski, J. Topp, W. Hansen, and D. Heitmann, Applied Physics Letters 93, 262501 (2008).

[8] F. Balhorn, S. Mansfeld, A. Krohn, J. Topp, W. Hansen, D. Heitmann, and S. Mendach, Physical Review Letters 104, 037205 (2010)

[9] M. Bauer, C. Mathieu, S. O. Demokritov, B. Hillebrands, P. A. Kolodin, S. Sure, H. Dötsch, V. Grimalsky, Y. Rapoport, and A. N. Slavin, Phys. Rev. B 56, R8483 
(1997)

[10] M. Bauer, O. Büttner, S. Demokritov, B. Hillebrands, V. Grimalsky, Y. Rapoport, and A. Slavin, Physical Review Letters 81, 3769 (1998).

[11] V. E. Demidov, S. O. Demokritov, K. Rott, P. Krzysteczko, and G. Reiss, Applied Physics Letters 91, 252504 (2007).

[12] J. Topp, J. Podbielski, D. Heitmann, and D. Grundler, Physical Review B 78, 024431 (2008).

[13] V. Demidov, S. Demokritov, K. Rott, P. Krzysteczko, and G. Reiss, Physical Review B 77, 064406 (2008).

[14] V. Demidov, J. Jersch, S. Demokritov, K. Rott, P. Krzysteczko, and G. Reiss, Physical Review B 79, 054417 (2009)

[15] O. Büttner, M. Bauer, S. O. Demokritov, B. Hillebrands, Y. S. Kivshar, V. Grimalsky, Y. Rapoport, and A. N. Slavin, Phys. Rev. B 61, 11576 (2000).

[16] V. E. Demidov, B. Hillebrands, S. O. Demokritov, M. Laufenberg, and P. P. Freitas, Journal of Applied Physics 97, 10A717 (2005).

[17] K. Perzlmaier, G. Woltersdorf, and C. Back, Physical Review B 77, 054425 (2008)

[18] V. Veerakumar and R. E. Camley, Phys. Rev. B 74,
$214401(2006)$

[19] V. E. Demidov, S. O. Demokritov, D. Birt, B. O'Gorman, M. Tsoi, and X. Li, Phys. Rev. B 80, 014429 (2009)

[20] T. Schneider, A. A. Serga, A. V. Chumak, C. W. Sandweg, S. Trudel, S. Wolff, M. P. Kostylev, V. S. Tiberkevich, A. N. Slavin, and B. Hillebrands, Phys. Rev. Lett. 104, 197203 (2010)

[21] A. A. Kostylev, M. P. Serga and B. Hillebrands, Physics Review Letters 106, 134101 (2011).

[22] M. R. Freeman and J. F. Smyth, Journal of Applied Physics 79, 5898 (1996).

[23] S. Tamaru, J. Bain, R. van De Veerdonk, T. Crawford, M. Covington, and M. Kryder, Physical Review B 70, 104416 (2004)

[24] E. K. Abbe, Archiv fuer Mikroskopische Anatomie 9, 413 (1873).

[25] H. X. Y. S. C. Liu, Z. Lee and X. Zhang, Science 315, 1686 (2007).

[26] S. Schwaiger, M. Bröll, A. Krohn, A. Stemmann, C. Heyn, Y. Stark, D. Stickler, D. Heitmann, and S. Mendach, Physical Review Letters 102, 163903 (2009).

[27] K. Guslienko, R. Chantrell, and A. Slavin, Physical Review B 68, 024422 (2003) 